\title{
\begin{tabular}{l|l} 
pcori). & PATIENT-CENTERED OUTCOMES RESEARCH INSTITUTE \\
RESEARCH SUMMARY
\end{tabular}
}

\section{Does Helping Patients with Cancer Manage Their Symptoms at Home Improve Their Quality of Life?}

Principal investigator

Susan McMillan, NP, PhD
Organization

University of South Florida

\section{What was the research about?}

Patients with cancer often have pain, feel tired, or have other symptoms. These symptoms can be severe or get in the way of daily activities, and negatively affect patients' lives.

The research team wanted to learn if a program for managing symptoms at home, called COPE, helped people with cancer have better quality of life, feel less depressed and anxious, and improve symptoms. In COPE, patients learned specific problem-solving skills. They met with a trained staff person three times and had two follow-up phone calls. Patients also watched a video and received written information. The team asked patients to keep a symptom diary.

The research team compared a group of patients who had usual care plus the COPE program with a group who had usual care alone and a group who had usual care plus extra support. With usual care, nurses taught patients how to manage their symptoms and whom to call for help. Patients in the group that got extra support had the same number of meetings and calls with trained staff as patients in COPE, but they didn't learn the skills in the COPE program.

\section{What were the results?}

Patients in COPE didn't have better quality of life or feel less depressed or anxious than patients in the other two groups. The intensity of symptoms decreased more for patients in COPE than patients who had usual care alone, but it was about the same for patients in COPE and patients who had extra support without learning the COPE program. How much patients' symptoms bothered them and patients' confidence in managing symptoms didn't differ among the three groups.

\section{Who was in the study?}

The study included 534 patients at a cancer treatment center in Florida. Of these, 89 percent were white. The average age was 58, and 57 percent were women. Patients were starting cancer treatment and had symptoms that upset them or that got in the way of their daily activities.

\section{What did the research team do?}

The research team assigned patients to one of the three groups by chance. The team collected study information in person and by phone when the study began, and then every week for 10 weeks. The team asked patients about their quality of life, feelings of depression and anxiety, and symptoms.

A group of patients reviewed the COPE program guide and video and gave input during the study.

\section{What were the limits of the study?}

This study didn't look at how well patients understood or remembered the COPE program. The program may not have worked as expected if people didn't find the information useful or memorable. Most patients in this study were white and were receiving care at one cancer center. Results might differ in other locations or with other patients. 
Future studies could examine other programs to help people with cancer manage symptoms at home.

Researchers could also look at ways to make the COPE program more helpful for patients.

\section{How can people use the results?}

Cancer treatment centers can use these results when looking at ways to help patients manage symptoms at home.

To learn more about this project, visit www.pcori.org/McMillan176. 